\title{
The Effect of Gravel Content on Fluidity and Mechanical Properties of Reactive Powder Concrete
}

\author{
Ding yahong ${ }^{1,2, *}, W_{u}$ jun $^{1}$, Xu haibin ${ }^{1}$ and Long baobin ${ }^{1}$ \\ ${ }^{1}$ School of Civil Engineering, Henan Polytechnic University, Jiaozuo 454003, China \\ ${ }^{2}$ International Joint Laboratory of Underground Space Development and Induced Disaster Prevention, Henan Polytechnic University, \\ Jiaozuo 454003, China
}

Received 2 October 2018; Accepted 23 December 2018

\begin{abstract}
$\overline{\text { Abstract }}$
Reactive powder concrete (RPC) is one kind of ultra-high performance concrete (UHPC), which has ultra-high strength, high toughness and high durability. However, its high cost and specific curing regime limit its extensive application. To reduce cost and simplify curing regime, cement-silica fume-fly ash ternary cementitious system, natural medium sand with well gradation for totally replacing the quartz sand and normal curing regime for specimens were used. Then six types of mix proportions for RPC were designed, and the gravel content that replaced natural medium sand were $0 \%$, $20 \%, 25 \%, 30 \%, 35 \%$ and $40 \%$, respectively. The fluidity and mechanical properties of the specimens, such as compressive, flexural, and splitting tensile strength, were investigated. Results show that the fluidity always increase with the gravel content and slump-flow is more apparent than that of the slump, while the compressive, flexural, and splitting tensile strength increase until reach the peak value when the gravel content is about $35 \%$, and these strengths are $20 \%$, $90 \%$ and $65 \%$ higher than that of RPC without gravel, respectively. Therefore, RPC incorporated with gravel can not only improve its fluidity and mechanical properties, but also reduce the engineering cost. The conclusions obtained in the study are of important theoretical value to direct the similar engineering practice.
\end{abstract}

Keywords: Reactive powder concrete, Gravel content, Fluidity, Mechanical properties

\section{Introduction}

Reactive powder concrete (RPC) is one kind of ultra-high performance concrete (UHPC) [1,2], and with high strength concrete (HSC) and high performance concrete (HPC), this new cement-based composite was developed firstly by Pierre Richard and Marcel Cheyrezy who came from BOUYGUES company in French in 1993, which has ultra-high strength, high toughness and high durability. According to its composition, the applied pressure and heating temperature in curing process, RPC can be divided into RPC200 and RPC800 [3].

RPC is composed of active powder materials such as cement and mineral admixtures, fine aggregates, additives, high-strength steel fibres and/or organic synthetic fibres and water. After homogeneously mixing, it is set and hardened through heat-pressing curing process. The emergence of RPC had attracted extensive attentions relying on its performance advantages in many aspects, and it had been gradually applied in many engineering fields, such as bridge engineering, municipal engineering, building engineering, nuclear power engineering, military engineering, and so on. For example, the single-span pedestrian/bikeway bridge, which had been erected in July 1997 in Sherbrooke, Quebec Province, Canada, is the world's first major structure to be built with RPC [4, 5]. Lafarge company built Mars Hill single-span bridge using RPC in Iowa State, USA, which was described as the The Future Bridge [6]. Nanjing Air Force Design Office in China cooperated with Shanghai

*E-mail address: dingyahong@hpu.edu.cn

ISSN: $1791-2377$ @ 2018 Eastern Macedonia and Thrace Institute of Technology. All rights reserved. doi:10.25103/jestr.116.21
Metro Corporation, had studied the application of RPC in air defense works of metro, and formulated the local standard of RPC protection door for air defense works [7].

Although lots of scholars around the world have carried out extensive, systematic and in-depth research on various properties of RPC, its high engineering cost and specific curing regime still limit its extensive application.

\section{State of the art}

Recently, studies on RPC have been mainly focused on the following aspects: mix proportion, static and dynamic mechanical properties, high temperature residual strength and spalling properties, toughening and fracture properties, hydration dynamics and microstructure, durability.

Han et al. used the short-cut super-fine stainless wire (SSSW) with super-fine diameter and high aspect ratio to reinforce RPC, and the calculation models of flexural strength andtoughness of SSSW RPC were established [8]. Ruan et al. investigated the effects of four types of multiwalled carbon nanotubes (MWCNT) on the mechanical properties of RPC and they found that adding proper type and content of MWCNT could effectively improve mechanical properties of RPC [9]. Yiğiter et al. used class-C fly ash (FA) to replace cement with a high-volume rate up to $60 \%$, and the results showed that compressive strength of $200 \mathrm{MPa}$ could be reached [10]. Liu et al. carried out experimental and numerical studies on impact resistance of RPC targets reinforced with 44-layer steel wire meshes, which indicated an effective impact resistance in comparison with the previous studies on UHPC with additions of fibres 
and basalt aggregates [11]. Yi et al. performed ANFO blast tests on reinforced UHSC and RPC panels, and they found that UHSC and RPC have better blast explosion resistance than normal strength concrete [12]. Ju et al. conducted numerical and experimental study on the thermal spalling mechanism of RPC exposed to high temperature, and they found that the numerical simulation was in good agreement with the experimental observations [13]. Hou et al. studied the effect of fire insulation on fire resistance of hybrid-fibre reinforced RPC beams, the post-fire test observation reveals that no fire-induced spalling occurred [14].

İpek et al. examined the effect of pre-setting pressure on RPC, and the maximum flexural strength of $36.40 \mathrm{MPa}$ and three times toughness of that were obtained under $25 \mathrm{MPa}$ pre-setting pressure, with the volume of sample decreased $7.9 \%$ [15]. Bonneau et al. studied the hydration kinetic of RPC by using electrical conductivity and isothermal calorimetry, they obtained a linear relationship between the logarithm of conductivity and the degree of hydration and proposed a continuous determination of the degree of hydration based on electrical conductivity [16]. Tam et al. examined the influences of water-to-binder ratio and superplasticizer dosage on the drying shrinkage and water permeability of RPC, several recommendations were given to reduce drying shrinkage and water permeability of RPC [17]. Tao et al. investigated the effect of steel fibre content, sand-binder ratio and water-binder ratio on the cracking resistant behaviour of RPC by an orthogonal experiment, and they revealed that steel fibre content has the most significant effect [18]. Peng et al. produced RPC specimens whose content of phosphorous slag powder (PS) and silica fume was about $50 \%$ (by the weight of binder), and the freezethaw and sulphate resistance tests verified their excellent durability properties [19]. The related researches were also conducted by others [20-22].

Replacing fine aggregate with gravel plays a positive role in reducing engineering cost, however, there are few studies on replacing fine aggregate of RPC with gravel up to now. So the effect of gravel content on fluidity and mechanical properties of RPC were studied in this study.

The rest of this study is organized as follows: Section 3 describes materials and the test design. Section 4 analyzes the results and gives some discussions. Section 5 provides the relevant conclusions.

\section{Methodology}

\subsection{Materials}

The cement P.O 42.5 with density of $3.11 \mathrm{~g} / \mathrm{cm}^{3}$ was produced in Jiaozuo Jiangu Cement Co., Ltd, China. The characteristic parameters of fly ash and silicon fume are shown in Tables 1 and 2, respectively. The fine aggregate is natural river sand with fineness modulus of 2.7 and apparent density of $2.6 \mathrm{~g} / \mathrm{cm}^{3}$. The coarse aggregate is gravel with 5 $10 \mathrm{~mm}$ particle size and continuous gradation, and the mud content is less than $0.5 \%$ after washing. The form of steel fibres are shearing end profiled, with diameter of $0.22 \mathrm{~mm}$ and length of $13 \mathrm{~mm}$. The water reduction rate of polycarboxylate super-plasticizer is $30 \%$. Defoamer is DF849 type white powder and mixing water is tap water.

Table 1. The characteristic parameters of fly ash

\begin{tabular}{l|c}
\multicolumn{1}{c|}{ Item } & Value \\
\hline Density $\left(\mathrm{g} / \mathrm{cm}^{3}\right)$ & 2.88 \\
Bulk density $\left(\mathrm{g} / \mathrm{cm}^{3}\right)$ & 1.25 \\
Specific surface area $\left(\mathrm{cm}^{2} / \mathrm{g}\right)$ & 2100 \\
$\mathrm{SO}_{3}(\%)$ & 1.20 \\
$\mathrm{CaO}(\%)$ & 3.60 \\
Loss of ignition $(\%)$ & 1.02 \\
\hline
\end{tabular}

Table 2. The characteristic parameters of silicon fume

\begin{tabular}{l|c}
\multicolumn{1}{c|}{ Item } & Value \\
\hline Density $\left(\mathrm{g} / \mathrm{cm}^{3}\right)$ & 1.65 \\
Bulk density $\left(\mathrm{g} / \mathrm{cm}^{3}\right)$ & 0.69 \\
Average particle size $(\mu \mathrm{m})$ & 0.15 \\
Specific surface area $\left(\mathrm{cm}^{2} / \mathrm{g}\right)$ & 28 \\
$\mathrm{SiO}_{2}(\%)$ & $\geq 98$ \\
$\mathrm{Al}_{2} \mathrm{O}_{3}(\%)$ & $\leq 0.70$ \\
$\mathrm{Fe}_{3} \mathrm{O}_{4}(\%)$ & $\leq 0.60$ \\
$\mathrm{MgO}(\%)$ & $\leq 0.50$ \\
$\mathrm{CaO}(\%)$ & $\leq 0.20$ \\
\hline
\end{tabular}

\subsection{Experimental program}

\subsubsection{Mix proportions}

As shown in Table 3, according to the theory of maximum compactness and the principle of minimum water requirement, six types of mix proportions were designed and denoted as $\mathrm{S}_{1}, \mathrm{~S}_{2}, \mathrm{~S}_{3}, \mathrm{~S}_{4}, \mathrm{~S}_{5}$, and $\mathrm{S}_{6}$, respectively, in which the gravel 5-10 $\mathrm{mm}$ content replaced natural medium sand were $0 \%, 20 \%, 25 \%, 30 \%, 35 \%$ and $40 \%$, respectively.

Table 3. The mix proportions of RPC

\begin{tabular}{|c|c|c|c|c|c|c|c|c|c|}
\hline \multirow{2}{*}{$\begin{array}{c}\text { Specimen } \\
\text { No. }\end{array}$} & \multicolumn{9}{|c|}{ Amount $\left(\mathrm{kg} / \mathrm{m}^{3}\right)$} \\
\hline & Cement & Fly ash & Silicon fume & Medium sand & Gravel & Water & Super-plasticizer & Defoamer & Steel fibre \\
\hline $\begin{array}{l}\mathrm{S}_{1} \\
\mathrm{~S}_{2} \\
\mathrm{~S}_{3} \\
\mathrm{~S}_{4} \\
\mathrm{~S}_{5} \\
\mathrm{~S}_{6}\end{array}$ & 895 & 268 & 90 & $\begin{array}{l}895 \\
716 \\
671 \\
627 \\
582 \\
537\end{array}$ & $\begin{array}{c}0 \\
179 \\
224 \\
268 \\
313 \\
358\end{array}$ & 250 & 15 & 3.759 & 156 \\
\hline
\end{tabular}

Note: The content of super-plasticizer was $1.2 \%$ of the cementitious materials and that of defoamer was $0.3 \%$ by mass, while that of steel fibre was $2 \%$ of those by volume.

Table 4. The test items and the requirements for specimens

\begin{tabular}{c|c|c}
\hline Test items & Amount (Group) & Size (mm $\times$ mm $\times$ mm) \\
\hline Compressive strength & 9 & $100 \times 100 \times 100$ \\
Flexure strength & 3 & $100 \times 100 \times 400$ \\
Splitting tensile strength & 3 & $100 \times 100 \times 100$ \\
\hline
\end{tabular}

Note: The compressive strength was tested for 7 days, 14 days and 28 days, respectively.

\subsubsection{Specimens preparation}

The test requirements for specimens are shown in Table 4. The specimens were produced by uniaxial forced mixer and the procedure was as follows: (1) To place natural medium sand, gravel and steel fibres into the mixer, and it were mixed for 2 minutes. (2) To place cement, fly ash and silicon 
fume into the mixer, and it were mixed for 2 minutes. (3) To place water (with super-plasticizer and defoamer dissolved in) slowly into the mixer, and it was mixed for 5 minutes.

The casting of specimens and the measurement of the rheological properties of the RPC were commenced as soon as mixing being completed. All the specimens were placed on a vibrating table and remained for 1 minute after filling, and then were moved to the standard curing room with its exposed surface being covered in plastic sheeting to prevent moisture evaporation. The specimens were demoulded after casting for 48 hours, and then sprinkled water on the specimens every morning until 28 days.

\subsubsection{Test program}

The fluidity of the RPC which included slump and slumpflow were tested according to reactive powder concrete in China (GB/T 31387-2015), as shown in Fig. 1.

The compressive strength were tested by electronic hydraulic testing machine with a $1000 \mathrm{kN}$ capacity at a loading rate of $1.20-1.40 \mathrm{MPa} / \mathrm{s}$ according to reactive powder concrete in China (GB/T 31387-2015), the compressive strength setup was shown in Fig. 2.

The splitting tensile strength were tested by the electronic hydraulic testing machine at a loading rate of $0.08-0.10 \mathrm{MPa} / \mathrm{s}$ according to standard for test method of mechanical prosperities on ordinary concrete in China $(\mathrm{GB} / \mathrm{T}$ 50081-2002), the splitting tensile strength test was shown in Fig. 3.

The flexural strength were tested by universal testing machine with a $100 \mathrm{kN}$ capacity at a loading rate of 0.08 $0.10 \mathrm{MPa} / \mathrm{s}$ according to GB/T 31387-2015 in China. Fourpoint bending was used in the test and the distance between the two supports was divided into three equal parts by the two loading points, the flexural strength setup was shown in Fig. 4.

\section{Results and Discussion}

The tests results were listed in Table 5.

Table. 5. The test results

\begin{tabular}{|c|c|c|c|c|c|c|c|}
\hline \multirow{2}{*}{ specimen } & \multicolumn{2}{|c|}{ Fluidity (mm) } & \multicolumn{3}{|c|}{ Compressive strength (MPa) } & \multirow{2}{*}{$\begin{array}{c}\text { Flexure strength } \\
\text { (MPa) }\end{array}$} & \multirow{2}{*}{$\begin{array}{c}\text { Split tensile strength } \\
\text { (MPa) }\end{array}$} \\
\hline & Slump & Slump-flow & $7 d$ & 14d & $28 d$ & & \\
\hline $\mathrm{S}_{1}$ & 220 & 400 & 74.2 & 82.8 & 97.7 & 8.26 & 9.08 \\
\hline $\mathrm{S}_{2}$ & 240 & 440 & 75.3 & 83.3 & 96.1 & 12.37 & 11.75 \\
\hline $\mathrm{S}_{3}$ & 250 & 510 & 78.8 & 86.5 & 102.6 & 13.54 & 14.53 \\
\hline $\mathrm{S}_{4}$ & 260 & 550 & 79.7 & 86.2 & 107.6 & 14.36 & 14.44 \\
\hline $\mathrm{S}_{5}$ & 290 & 580 & 82.7 & 92.1 & 116.5 & 15.71 & 15.02 \\
\hline $\mathrm{S}_{6}$ & 330 & 650 & 73.6 & 81.4 & 104.8 & 10.78 & 12.59 \\
\hline
\end{tabular}

\subsection{Fluidity of fresh RPC}

Fig. 5 showed the change trends of fluidity of fresh RPC with the gravel content increasing, it could be seen that the fluidity always increased with the gravel content and slumpflow, which was more apparent than that of the slump. When the gravel content exceeded $35 \%$, the segregation of RPC occurred, the reasons could be explained as follows: firstly, the binder played a lubricant and roll-bead role in aggregates. Secondly, the total specific surface area decreased with the increase of the gravel content because of the gravel content was small than that of sand. Thus, considering the two reasons mentioned above, there would be redundant binder to cover the surface of the aggregates, which could cause the segregation of fresh RPC. Therefore, a reasonable sand rate

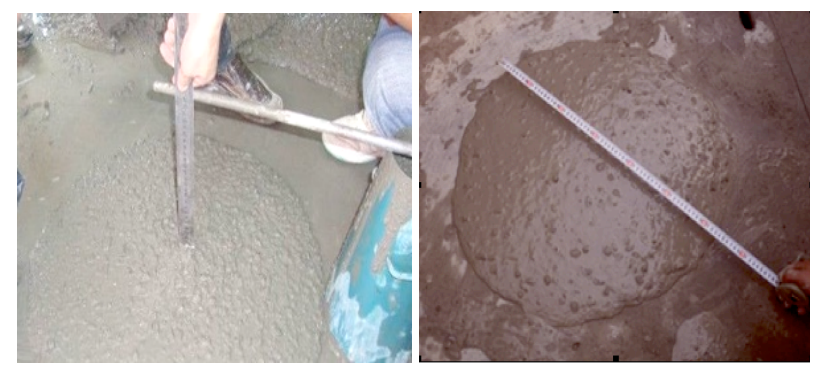

Fig. 1. Slump and slump-flow

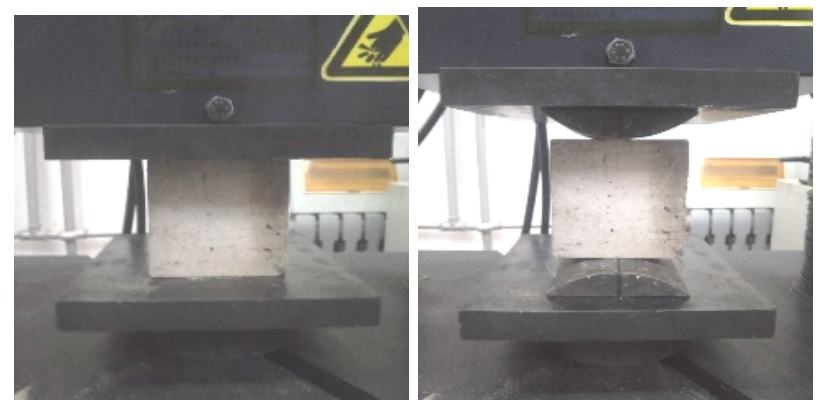

Fig. 2. Compressive strength test Fig. 3. Splitting tensile strength test

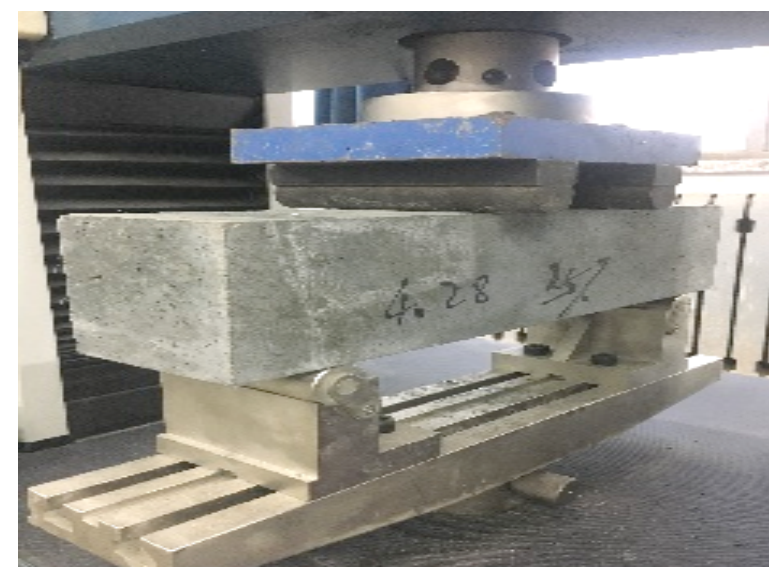

Fig. 4. Flexural strength setup was one of the key factors determining the properties of RPC.

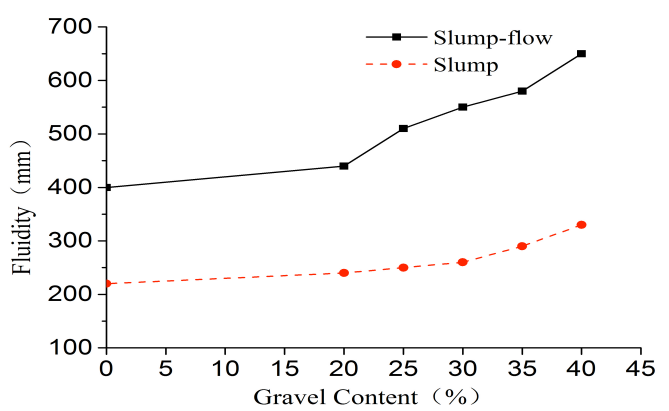

Fig. 5. Fluidity of fresh RPC 


\subsection{Compressive strength of RPC}

Fig. 6 showed the change trends of compressive strength of RPC with the gravel content increasing, we found that the compressive strength of RPC increased at first and then decreased, and it reached the peak value when the gravel content was about $35 \%$, the strength was $11 \%$ and $20 \%$ higher than that of RPC without gravel at 7 days and 28 days, respectively.

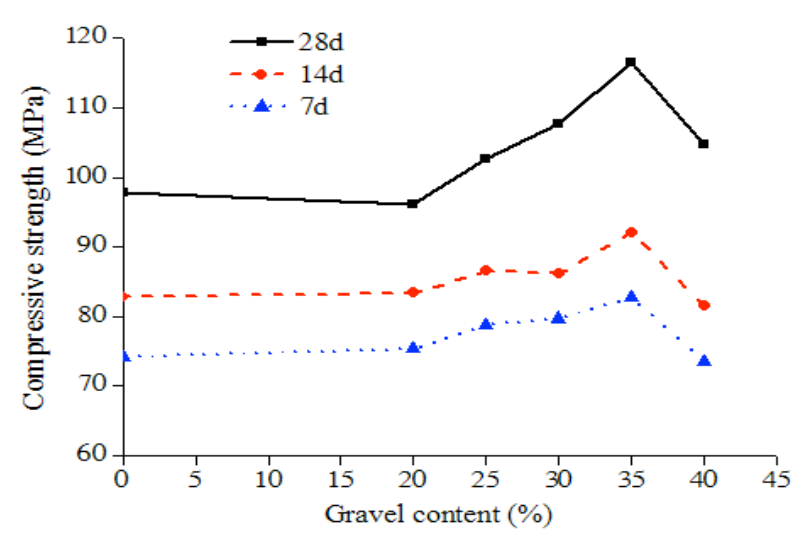

Fig. 6. Compressive strength of RPC

The incorporation of gravel played a skeleton role in RPC which can enhance its compressive strength. However, as mentioned above in 4.1 part, when the gravel content exceeded $35 \%$, there would be redundant binder to cover the surface of the aggregates, which could cause the segregation of fresh RPC and decreased compressive strength of RPC. Hence, considering the interaction of the two reasons mentioned above, the optimum gravel content was about $35 \%$ for the compressive strength of RPC.

\subsection{Flexural strength of RPC}

Fig. 7 illustrated the relationship between the flexural strength of RPC and the gravel content, we found that the flexural strength of RPC increased roughly linearly at first and then decreased, and it reached its peak value when the gravel content was about $35 \%$, which was $90 \%$ higher than that of RPC without gravel.

As mentioned above in 4.2 part, it was effected by the interaction of the segregation of fresh RPC and the skeleton role in RPC, the optimum gravel content was about 35\% for the flexural strength of RPC. Through the linear fitting, the relationship expression for the roughly linear growth part with the gravel content of RPC showed as follows, $y=0.21 x+8.22$, whose correlation coefficient $\mathrm{R}^{2}$ was 0.95 .

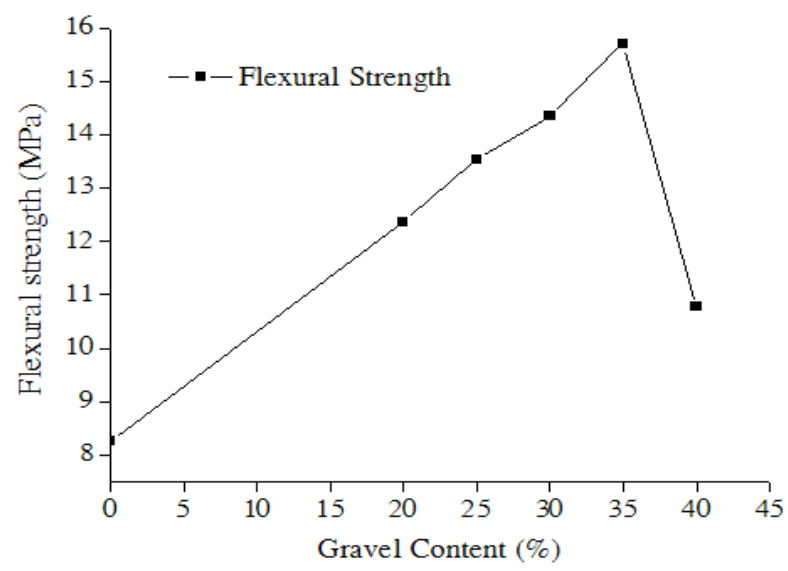

Fig. 7. Flexural strength of RPC

\subsection{Splitting tensile strength of RPC}

Fig. 8 illustrated the relationship between the splitting tensile strength of RPC and the gravel content, we found that the flexural strength of RPC increased approximately at first and then decreased, and it reached its peak value when the gravel content was about $35 \%$, which was $65 \%$ higher than that of RPC without gravel.

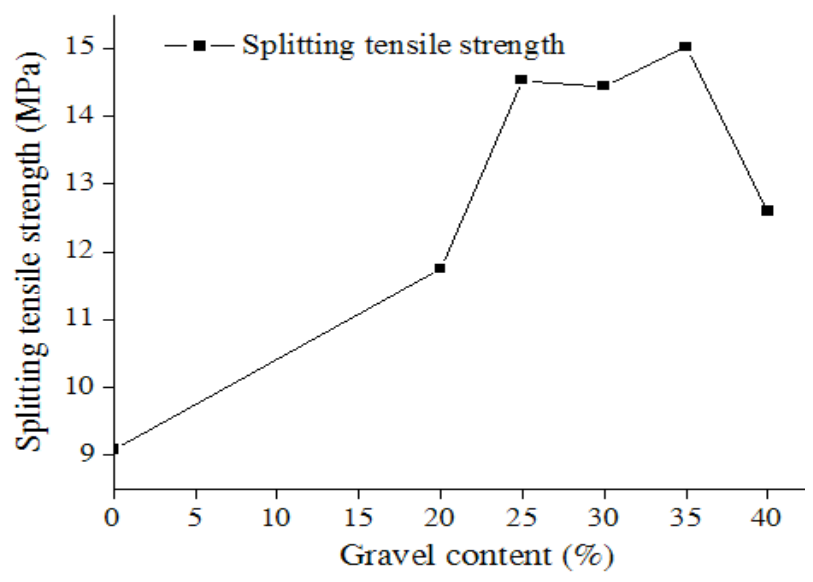

Fig. 8. Splitting tensile strength of RPC

As mentioned above in 4.2 part, it was effected by the interaction of the segregation of fresh RPC and the skeleton role in RPC, the optimum gravel content was $35 \%$ for the splitting tensile strength of RPC.

\section{Conclusions}

To study the effect of gravel content on fluidity and mechanical properties of reactive powder concrete, some mechanical tests of RPC specimens were conducted, the following conclusions were obtained:

(1) The fluidity always increased with the gravel content and slump-flow was more apparent than that of the slum. When the gravel content exceeded $35 \%$, the segregation of RPC occurred. Therefore, a reasonable sand rate was one of the key factors determining the properties of RPC.

(2) The mechanical properties of the specimens, such as compressive, flexural, and splitting tensile strength increased until the peak values when the gravel content was about $35 \%$, the strengths were $20 \%, 90 \%$ and $65 \%$ higher than that of RPC without gravel, respectively.

(3) Through the linear fitting, the relationship expression for the roughly linear growth part with the gravel content of flexural strength of RPC showed as follows, $y=0.21 x+8.22$, whose correlation coefficient $\mathrm{R}^{2}$ was 0.95

RPC incorporated with gravel can not only improve its fluidity and mechanical properties, but also reduce the engineering cost. The research results can provide references for the similar engineering practice.

\section{Acknowledgements}

The study was supported by the National Natural Science Fund of China (51108161) and Henan Provincial Department of Transportation (2016Y2-1).

This is an Open Access article distributed under the terms of the Creative Commons Attribution License 


\section{References}

1. Matte, V., Moranville, M., Adenot, F., Richet, C., Torrenti, J. M. "Simulated microstructure and transport properties of ultra-high performance cement-based materials". Cement and Concrete Research, 30(12), 2000, pp. 1947-1954.

2. Rahman, S., Molyneaux, T., Patnaikuni, I. "Ultra high performance concrete: Recent applications and research". Australian Journal of Civil Engineering, 2(1), 2005, pp. 13-20.

3. Richard, P., Cheyrezy, M. "Composition of reactive powder concretes". Cement and Concrete Research, 25(7), 1995, pp. 15011511.

4. Blais, P. Y., Couture, M. "Precast, prestressed pedestrian bridge world's first reactive powder concrete structure". PCI Journal, 44(5), 1999, pp. 60-71.

5. AÏtcin, P., Lachemi, M., Adeline, R., Richard, P. "The sherbrooke reactive powder concrete footbridge". Structural Engineering International, 8(2), 1998, pp. 140-144.

6. Liu, S., Yan, P., Feng, J. "Progress on research and application of reactive powder concrete (RPC)". National Academic Symposium on Special Concrete Technology and Engineering Application and 2008 Annual Meeting of Concrete Quality Professional Committee. Xi'an: 2008, pp. 113-117.

7. Yan, P. "The development and present statue of ultra-high performance concrete (UHPC)". Concrete World, (9), 2010, pp. 3641.

8. Han, B., Dong, S., Ou, J., Zhang, C., Wang, Y., Yu, X., Ding, S. "Microstructure related mechanical behaviors of short-cut superfine stainless wire reinforced reactive powder concrete". Materials \& Design, 96, 2016, pp. 16-26.

9. Ruan, Y., Han, B., Yu, X., Zhang, W., Wang, D. "Carbon nanotubes reinforced reactive powder concrete". Composites Part A, 112, 2018, pp. 371-382.

10. Yi iter, H., Ayd n, S., Yaz c , H., Yard mc , M. Y. "Mechanical performance of low cement reactive powder concrete (LCRPC". Composites Part B: Engineering, 43(8), 2012, pp. $2907-$ 2914.

11. Liu, J., Wu, C., Li, J., Su, Y., Shao, R., Liu, Z., Chen, G. "Experimental and numerical study of reactive powder concrete reinforced with steel wire mesh against projectile penetration". International Journal of Impact Engineering, 109, 2017, pp. 131149.
12. Yi, N., Kim, J. J., Han, T., Cho, Y., Lee, J. H. "Blast-resistant characteristics of ultra-high strength concrete and reactive powder concrete". Construction and Building Materials, 2012, 28(1), pp 694-707.

13. Ju, Y., Liu, J., Liu, H., Tian, K., Ge, Z. "On the thermal spalling mechanism of reactive powder concrete exposed to high temperature: Numerical and experimental studies". International Journal of Heat and Mass Transfer, 98, 2016, pp. 493-507.

14. Hou, X., Ren, P., Rong, Q., Zheng, W., Zhan, Y. "Effect of fire insulation on fire resistance of hybrid-fiber reinforced reactive powder concrete beams". Composite Structures, 209, 2019, pp. 219-232.

15. pek, M., Yilmaz, K., Uysal, M. "The effect of pre-setting pressure applied flexural strength and fracture toughness of reactive powder concrete during the setting phase". Construction and Building Materials, 26(1), 2012, pp. 459-465.

16. Bonneau, O., Vernet, C., Moranville, M., A ${ }^{\cdot}$ tcin, P. "Characterization of the granular packing and percolation threshold of reactive powder concrete". Cement and Concrete Research, 30(12), 2000, pp. 1861-1867.

17. Tam, C. M., Tam, V. W. Y., Ng, K. M. "Assessing drying shrinkage and water permeability of reactive powder concrete produced in Hong Kong”. Construction and Building Materials, 26(1), 2012, pp. 79-89.

18. Ji, T., Chen, C., Zhuang, Y. "Evaluation method for cracking resistant behavior of reactive powder concrete". Construction and Building Materials, 28(1), 2012, pp. 45-49.

19. Peng, Y., Zhang, J., Liu, J., Ke, J., Wang, F. "Properties and microstructure of reactive powder concrete having a high content of phosphorous slag powder and silica fume". Construction and Building Materials, 101, 2015, pp. 482-487.

20. Wang, S. R., Xiao, H. G., Hagan, P., Zou, Z. S., "Mechanical behavior of fully-grouted bolt in jointed rocks subjected to double shear tests". Dyna, 92(3), 2017, pp.314-320.

21. Zhang, X. G., Kuang, X. M., Yang, J. H., Wang, S. R., "Experimental study on mechanical properties of lightweight concrete with shale aggregate replaced partially by nature sand". Electronic Journal of Structural Engineering, 17(1), 2017, pp.85-94.

22. Wang, S. R., Xiao, H. G., Cao, C., Zou, Z S., Liu, X. L, "Simulation verification analysis of anchoring characteristics of transverse rib steel bar during pull-out test”. Dyna, 91(5), 2016, pp. 548-553. 